\title{
KASCADE-Grande: Composition studies in the view of the post-LHC hadronic interaction models
}

\author{
A. Haungs ${ }^{1, a, b}$, W.D. Apel ${ }^{1}$, J.C. Arteaga-Velázquez ${ }^{2}$, K. Bekk ${ }^{1}$, M. Bertaina ${ }^{3}$, J. Blümer ${ }^{1,4,14}$, H. Bozdog ${ }^{1}$, I.M. Brancus ${ }^{5}$, \\ E. Cantoni ${ }^{3,6,15}$, A. Chiavassa ${ }^{3}$, F. Cossavella ${ }^{4}, 16$, K. Daumiller ${ }^{1}$, V. de Souza ${ }^{7}$, F. Di Pierro ${ }^{3}$, P. Doll ${ }^{1}$, R. Engel ${ }^{1}$, \\ D. Fuhrmann ${ }^{8,17}$, A. Gherghel-Lascu ${ }^{5}$, H.J. Gils ${ }^{1}$, R. Glasstetter ${ }^{8}$, C. Grupen ${ }^{9}$, D. Heck ${ }^{1}$, J.R. Hörandel ${ }^{10}$, T. Huege ${ }^{1}$, \\ K.-H. Kampert ${ }^{8}$, D. Kang ${ }^{1}$, H.O. Klages ${ }^{1}$, K. Link ${ }^{4}$, P. Łuczak $^{11}$, H.J. Mathes ${ }^{1}$, H.J. Mayer ${ }^{1}$, J. Milke ${ }^{1}$, B. Mitrica ${ }^{5}$, \\ C. Morello ${ }^{6}$, J. Oehlschläger ${ }^{1}$, S. Ostapchenko ${ }^{12}$, T. Pierog ${ }^{1}$, H. Rebel ${ }^{1}$, M. Roth ${ }^{1}$, H. Schieler ${ }^{1}$, S. Schoo ${ }^{1}$, F.G. Schröder ${ }^{1}$, \\ O. Sima ${ }^{13}$, G. Toma ${ }^{5}$, G.C. Trinchero ${ }^{6}$, H. Ulrich ${ }^{1}$, A. Weindl ${ }^{1}$, J. Wochele ${ }^{1}$, and J. Zabierowski ${ }^{11}$
}

1 Institut für Kernphysik, KIT - Karlsruhe Institute of Technology, Germany

2 Universidad Michoacana, Inst. Física y Matemáticas, Morelia, Mexico

3 Dipartimento di Fisica, Università degli Studi di Torino, Italy

${ }^{4}$ Institut für Experimentelle Kernphysik, KIT - Karlsruhe Institute of Technology, Germany

5 Horia Hulubei National Institute of Physics and Nuclear Engineering, Bucharest, Romania

${ }^{6}$ Osservatorio Astrofisico di Torino, INAF Torino, Italy

7 Universidade São Paulo, Instituto de Física de São Carlos, Brasil

8 Fachbereich Physik, Universität Wuppertal, Germany

9 Department of Physics, Siegen University, Germany

${ }^{10}$ Dept. of Astrophysics, Radboud University Nijmegen, The Netherlands

11 National Centre for Nuclear Research, Department of Astrophysics, Lodz, Poland

12 Frankfurt Institute for Advanced Studies (FIAS), Frankfurt am Main, Germany

13 Department of Physics, University of Bucharest, Bucharest, Romania

14 now: Head of Division V at KIT - Karlsruhe Institute of Technology, Germany

15 now at: Istituto Nazionale di Ricerca Metrologia, INRIM, Torino

16 now at: DLR Oberpfaffenhofen, Germany

17 now at: University of Duisburg-Essen, Duisburg, Germany

\begin{abstract}
The KASCADE-Grande experiment has significantly contributed to the current knowledge about the energy spectrum and composition of cosmic rays for energies between the knee and the ankle. Meanwhile, post-LHC versions of the hadronic interaction models are available and used to interpret the entire data set of KASCADE-Grande. In addition, a new, combined analysis of both arrays, KASCADE and Grande, was developed significantly increasing the accuracy of the shower observables. First results of the new analysis with the entire data set of the KASCADE-Grande experiment will be the focus of this contribution.
\end{abstract}

\section{KASCADE-Grande}

The Extensive Air Shower (EAS) experiment and cosmicray facility KASCADE finally stopped the active data acquisition end of 2012 after 20 years of measurements. KASCADE, its extension KASCADE-Grande as well as the radio test experiments LOPES and CROME are meanwhile fully decommissioned. The collaboration, however, continues the detailed analysis of the high-quality air-shower data. In addition, with KCDC, the KASCADE Cosmic-ray Data Center, we provide to the public the scientific data via a customized web page.

The multi-detector experiment KASCADE [1] (located in south-west Germany in the beautiful valley of the river Rhine at $49.1^{\circ} \mathrm{N}, 8.4^{\circ} \mathrm{E}, 110 \mathrm{~m}$ a.s.l.) started operation in 1993 and was extended to KASCADE-Grande in

\footnotetext{
a e-mail: haungs@kit.edu

b Speaker for the KASCADE-Grande Collaboration.
}

2003. Main parts of the facility were the Grande [2] array spread over an area of $700 \times 700 \mathrm{~m}^{2}$, the original KASCADE array covering $200 \times 200 \mathrm{~m}^{2}$ with unshielded and shielded detectors, a large-size hadron calorimeter, and additional muon tracking devices. The radio antenna field LOPES $[3,4]$ and the microwave experiment CROME $[5,6]$ were also important components of the experimental set-up of KASCADE-Grande. The full facility was in operation until end of 2012.

The main goal of the measurements was the estimation of energy and mass of the primary particles in a wide energy range. The analysis is based on the combined investigation of the charged particle, the electron, and the muon components measured by the detector arrays of Grande and KASCADE. The general idea of the data analyses of the KASCADE and KASCADE-Grande experiments is the determination of the chemical composition in the primary energy range $10^{14}-10^{18} \mathrm{eV}$ by reconstructing individual mass group spectra. Structures observed in these individual 


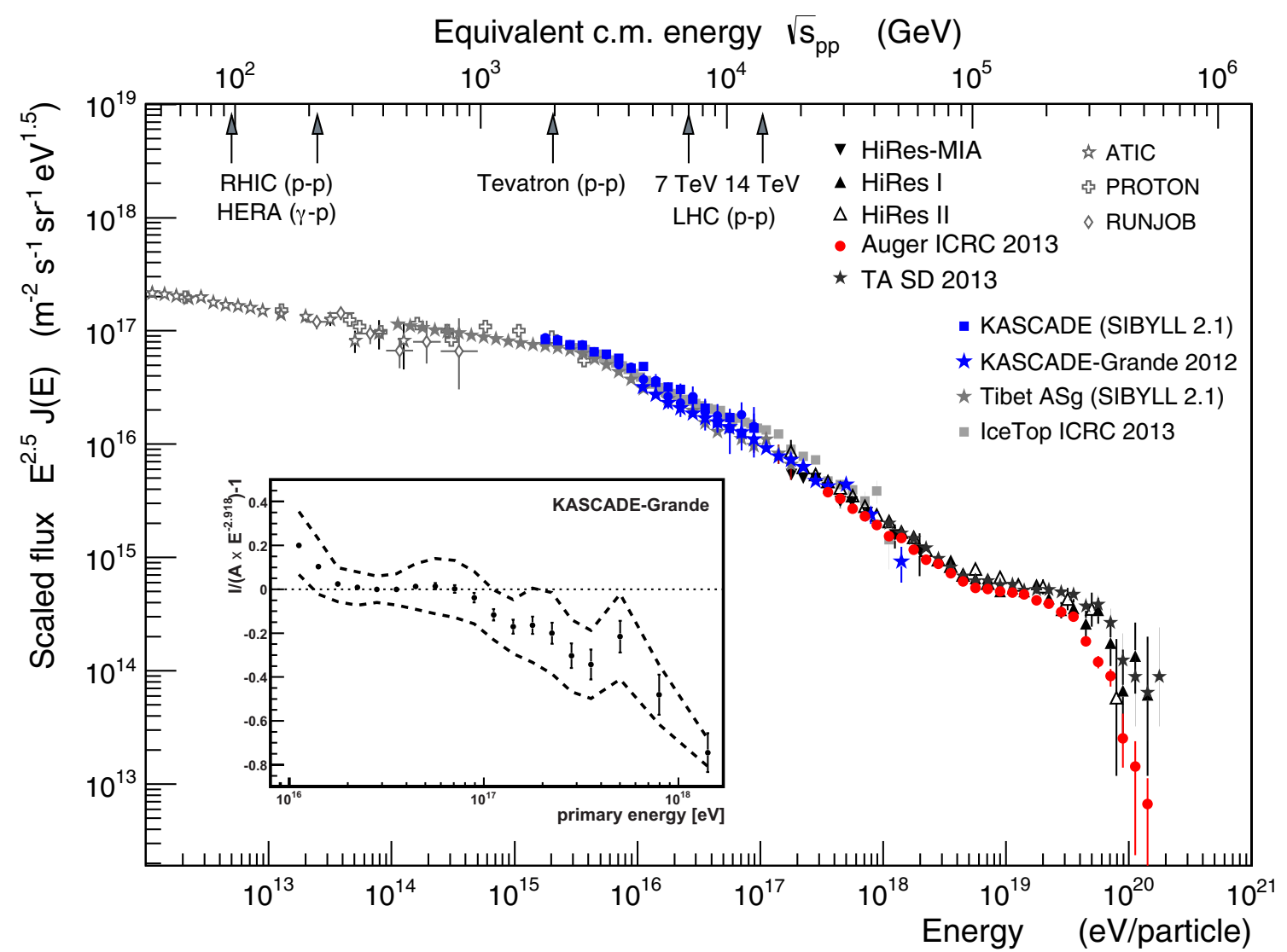

Figure 1. The all-particle energy spectrum [7] obtained with KASCADE and KASCADE-Grande (based on the QGSJet-II model and unfolded, i.e. corrected for the reconstruction uncertainties). Shown are the spectra in comparison with results of other experiments. In addition, the corresponding interaction energy at accelerators are indicated. The inset displays the spectrum of KASCADE-Grande from $10^{16} \mathrm{eV}$ to $10^{18} \mathrm{eV}$ (from reference [8]) in a way to better see the spectral features.

spectra provide strong constraints to astrophysical models of origin and propagation of high-energy cosmic rays.

\section{The all-particle energy spectrum}

By using the hadronic interaction model QGSJet-II as a baseline, a composition independent all-particle energy spectrum of cosmic rays was reconstructed in the energy range from $10^{16} \mathrm{eV}$ to $10^{18} \mathrm{eV}$ from the data of the Grande extension (using the Grande array for charge particle measurements and the KASCADE muon array detectors to reconstruct the low energy muon component) within a total uncertainty in flux of $10-15 \%$ [8]. The spectrum is in the overlapping region in agreement with the earlier published spectrum by KASCADE [9] $\left(10^{15} \mathrm{eV}\right.$ to $\left.10^{17} \mathrm{eV}\right)$. Significant structures are observed in the all-particle spectrum (Fig. 1): The justification of the 'knee' at a few times $10^{15} \mathrm{eV}$ is given since many years. In addition, with Grande, there is now clear evidence that just above $10^{16} \mathrm{eV}$ the spectrum shows a significant 'concave' behavior. A further feature in the spectrum is a small break, i.e. knee-like feature at around $10^{17} \mathrm{eV}$ (see inset of Fig. 1). Found first by KASCADEGrande this is meanwhile confirmed by other experiments (e.g., Tunka-133 [10] or IceTop [11]). This 'second knee' occurs at an energy where the rigidity dependent, i.e. charge dependent, knee of the iron component would be expected, if the 'first knee' is caused by light primaries. The concave part of the spectrum is then a consequence of knee-like features of the spectra of medium masses.
The co-location of LOPES with KASCADE-Grande as well as Tunka-Rex (the Tunka Radio extension) with Tunka-133 was used for an interesting cross-check of the absolute energy scale reconstructed by KASCADEGrande: The radio technique enables the reconstruction of the primary energy around $100 \mathrm{PeV}$ based on the amplitude of the radio signal. This amplitude can be measured absolutely, where the signal experiences no attenuation in the atmosphere. Due to a consistent amplitude calibration for Tunka-Rex and LOPES achieved by using the same reference source, the reconstruction is used to determine the air-shower energy on an absolute scale at both experiments. This was also used to compare the energy scales of the host experiments Tunka-133 and KASCADEGrande. As a result, it was confirmed that the energy scales of cosmic-ray measurements by the independently calibrated experiments KASCADE-Grande and Tunka-133 are consistent with each other on the level of $10 \%$ [12].

\section{Elemental composition of cosmic rays}

Already in 2005 KASCADE proved $[9,13]$ that the knee is caused by a decrease of the light mass group of primary particles and not by medium and heavy primary particles. With KASCADE-Grande we investigated such individual mass group spectra also at higher primary energies $[14,15]$. We performed all the simulations for this analysis with the air-shower simulation package CORSIKA [16] 


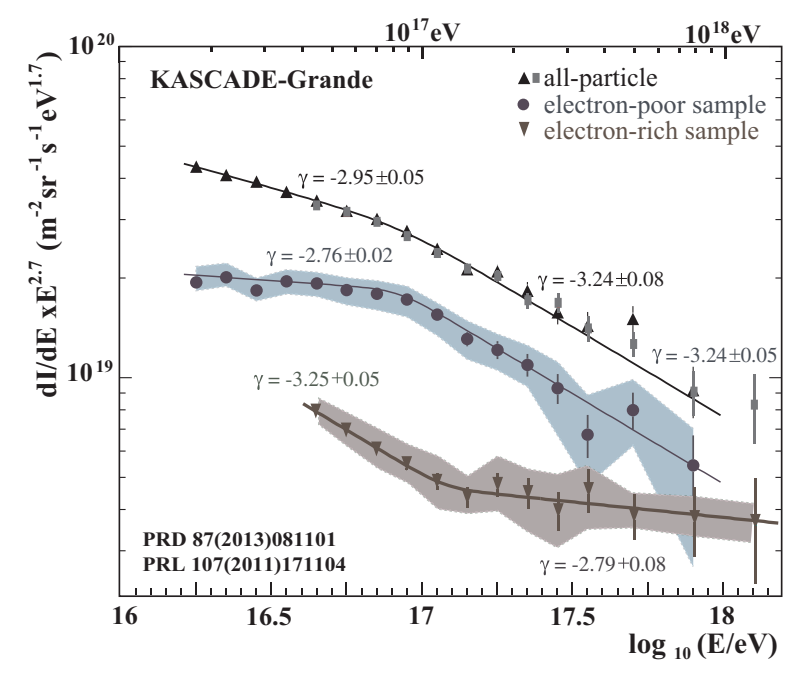

Figure 2. All-particle, electron-poor, and electron rich energy spectra from KASCADE-Grande [7]. References are given in the figure. All the results combined in this figure are based on the hadronic interaction models QGSJet-II-02 and FLUKA.

allowing simulations based on various hadronic interaction models. The application of this methodical approach to shower selection and separation in various mass groups was performed and cross-checked in different ways, where Fig. 2 shows the main results. In a first step we separated the Grande data in two mass groups only, i.e., in groups of heavy and light primary masses according to the electronmuon ratio in the air showers:

The reconstructed spectrum of the electron-poor events, i.e. the spectrum of heavy primaries, shows a distinct knee-like feature at about $8 \cdot 10^{16} \mathrm{eV}$ with a statistical significance of $3.5 \sigma[14,17]$. The change of the spectral slope is $\Delta \gamma=-0.48$ from $\gamma=-2.76 \pm$ 0.02 to $\gamma=-3.24 \pm 0.05$ with the break position at $\log _{10}(E / e V)=16.92 \pm 0.04$. The selection of heavy primaries enhances the knee-like feature that is already present in the all-particle spectrum. The analysis was repeated on the basis of different hadronic interaction models [18,19]. Despite the fact that the relative abundance of the heavy particles varies significantly dependent on the model in use, the spectral feature of this 'heavy' knee is visible in all the spectra. Figure 3 shows the results for the heavy primary spectra, but also for the light and all-particle spectra. It was found that the structures are similar for all models, but the relative abundances vary. This 'heavy' knee was confirmed by a detailed KASCADE-like unfolding analysis applied to the data of the Grande array [17]. This fits to a rapidity dependent knee scenario with the results of KASCADE [9], where a reduction of the light component beyond the first knee was observed. In addition, an ankle-like feature was observed in the spectrum of the electron-rich events (Fig. 2), i.e. light elements of the primary cosmic rays, at an energy of $10^{17.08 \pm 0.08} \mathrm{eV}$. At this energy, the spectral index changes by $\Delta \gamma=0.46$ with a statistical significance of $5.8 \sigma[15]$.

\section{Hadronic interaction models}

Historically, a great achievement of KASCADE was the invention of the CORSIKA air-shower simulation tool [16], which meanwhile is used by all major

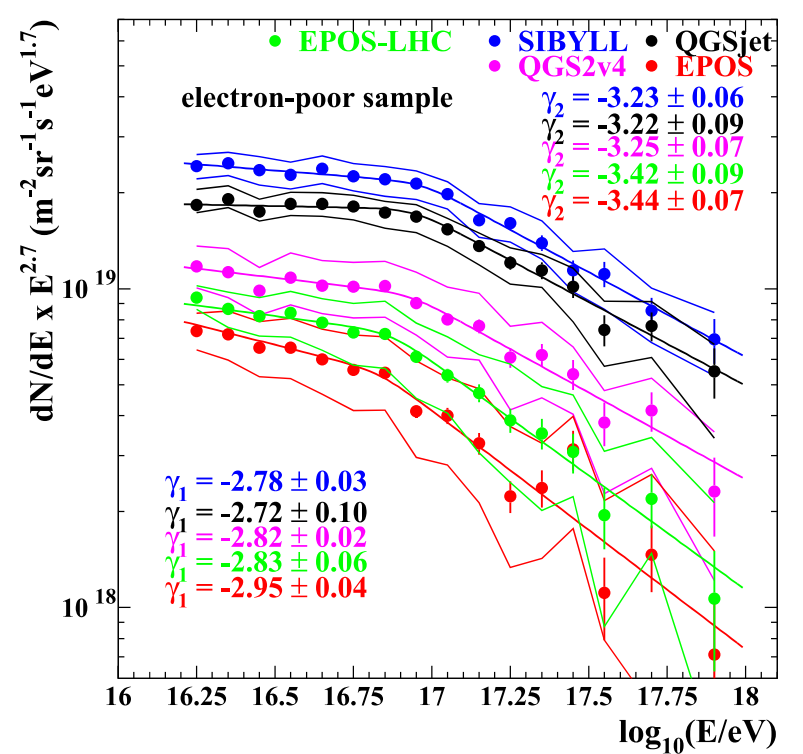

Figure 3. Reconstructed energy spectra of the heavy primary component for five hadronic interaction models. The error bars show the statistical uncertainties and the bands the systematic uncertainties; fits on the spectra and resulting slopes before and after the heavy knee are also indicated [19].

experiments worldwide. Mainly data of the hadron calorimeter and additional muon counters at the central detector and the muon tunnel were used within KASCADE to iteratively test and improve the various versions of the hadronic interaction models optionally available in CORSIKA (see e.g., $[13,20]$ and references therein).

Regarding the analysis of KASCADE and KASCADEGrande data one has to conclude that all versions of hadronic interaction models of the newest generation provide a 'physical' result in terms of energy and composition of primary cosmic rays. Here, physical means here that the mean composition lies within the band spanned by primary proton and iron simulations. However, the absolute energy and, in particular, the mass scale varies significantly from model to model. In addition, it varies partly also within the models if different shower observables taken from the electromagnetic, muonic, or hadronic components are used. This ambiguity can not be resolved by looking at one single observable or experiment, only. One approach to disentangle this puzzle is the combined analysis of data from the KASCADE and the KASCADE-Grande experiments.

\section{KASCADE and KASCADE-Grande combined analysis}

A specific analysis focuses on combining the KASCADE and KASCADE-Grande arrays already at the shower reconstruction. The number of charged particles and the number of muons is the result of a fit to the lateral densities measured by both arrays. Therefore, events located in the Grande array gain additional 252 density measurements and events located in the KASCADE array gain 37 additional measurements compared to the individual fits to the lateral densities (see Fig. 4). The additional stations at larger distances allow us to reach for KASCADE located events higher energies and for Grande located events a higher accuracy. 


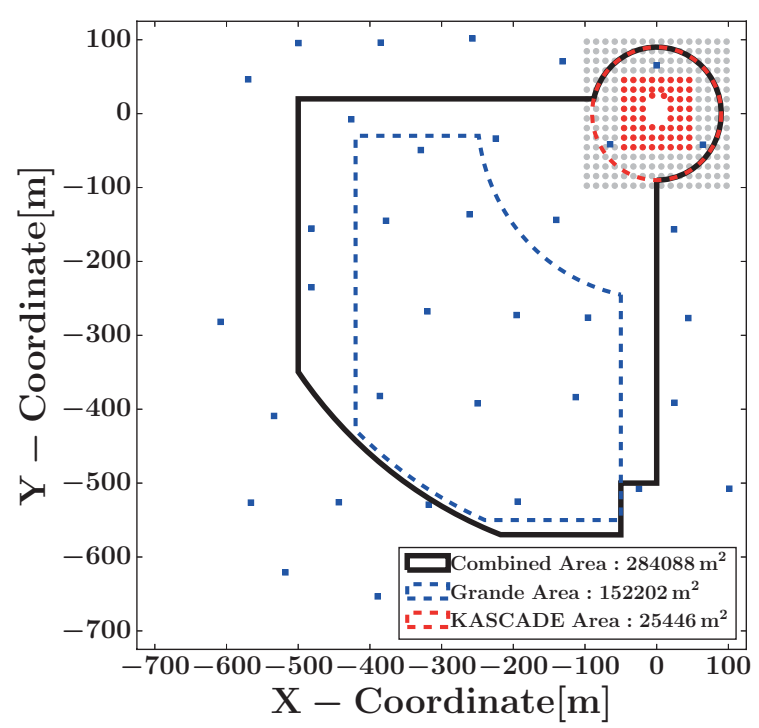

Figure 4. The stations of the KASCADE-Grande array (rectangles) are shown relative to the KASCADE array. In addition, the fiducial areas used for the two stand-alone reconstructions as well as the combined analysis are shown [21].

After converting the energy deposits to the number of particles at each detector using the methods of the stand-alone analyses but now for all detector stations of both arrays, the reconstruction procedure of the combined detector array follows the usual one: The number of particles corresponding to a certain energy deposited in a detector is calculated by dividing the total energy deposit by the effective energy deposited per single charged particle taking into account also the energy deposited by photons and the shower's radial dependent e $/ \gamma$ ratio. The electron size is obtained by fitting a NKG-like lateral density function to the measured particle densities. After this step, the shower size is the number of charged particles. The reconstruction of the number of muons is done simultaneously to the 192 muon detectors of the KASCADE array. Now, the muon Lateral Distribution Function (LDF) is included in the next iteration of the fitting procedure for the unabsorbed detectors resulting in the reconstruction of the shower size as the number of electrons. The reconstruction accuracies of the electron and muon shower size using the combined reconstruction, improves considerably compared to the stand alone analyses. The accuracy of the electron shower size at $10^{5}$ electrons improves from about $6 \%$ to about 4 to $5 \%$ for the combined reconstruction, reaching an accuracy of better than $3 \%$ towards higher energies.

The improvement in the muon reconstruction due to the combined use of both detectors is rather small at the threshold of full efficiency, where the number of muons is reconstructed with an accuracy improving from about $25 \%$ to $17 \%$. Therefore, the combined reconstruction reaches an improved accuracy and better uniformity over the entire range. The combination of the detectors right at the beginning of the reconstruction procedure results in much more accurately estimated observables. This is especially true for KASCADE-Grande, for which an improvement of several percent is achieved.

The new 2-dimensional shower size spectrum (Fig. 5) is then the object of further analysis. The distribution

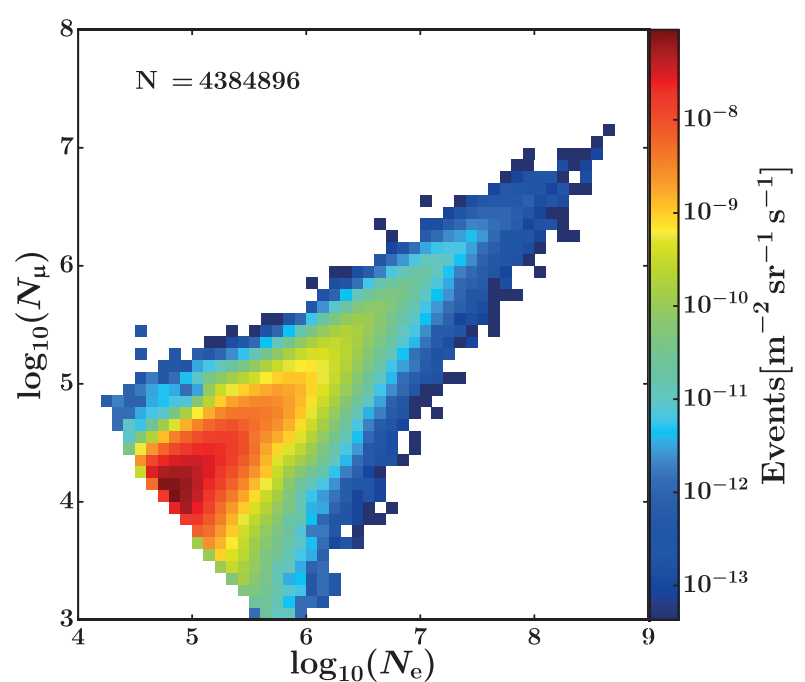

Figure 5. The two-dimensional shower size spectrum for KASCADE-Grande data obtained by a combined shower reconstruction of data from the KASCADE and the Grande arrays [21].

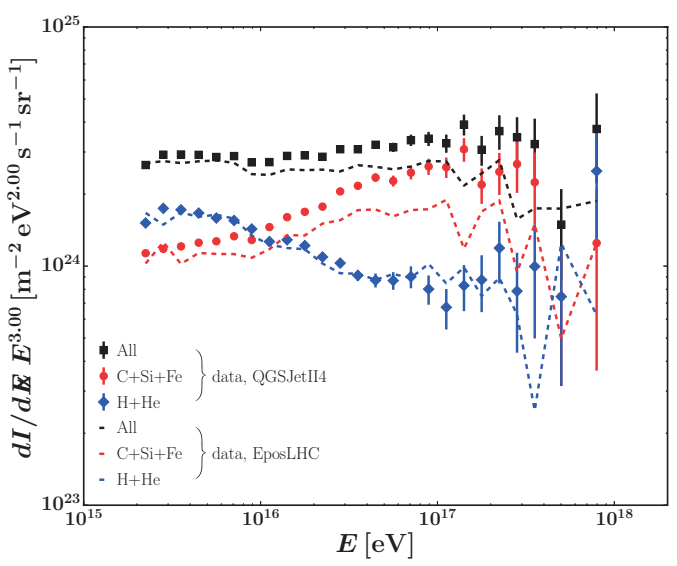

Figure 6. It is worth noting that all spectra obtained are labeled as 'RAW', which means that they have not been corrected for detector and reconstruction effects.

contains events from a larger fiducial area, are reconstructed with higher accuracy, and cover more than three orders of magnitude in primary energy. This plot contains $4,384,896$ reconstructed events. The final goal of the analysis is to obtain from this event sample the allparticle as well as mass group spectra by one methodically consistent reconstruction procedure [21].

As a first step, the method from the Grande stand alone data analysis (k-parameter method, where $\mathrm{k}$ is basically the electron-muon ratio of the individual showers taking into account the incident zenith angle) was applied to the new reconstructed two-dimensional shower size distribution to obtain the all-particle energy spectrum, as well as the spectra of the heavy- and light-induced air showers. For the calibration of the energy and mass of the primary particle a set of simulations is needed which is obtained for each hadronic interaction model, separately. The same hadronic interaction model was always used to define the separation value (the cut in the parameter ' $\mathrm{k}$ ') and to interpret the full data set.

Figure 6 shows the results of this analysis based on QGSJet-II-04 and EPOS-LHC for comparisons. The 


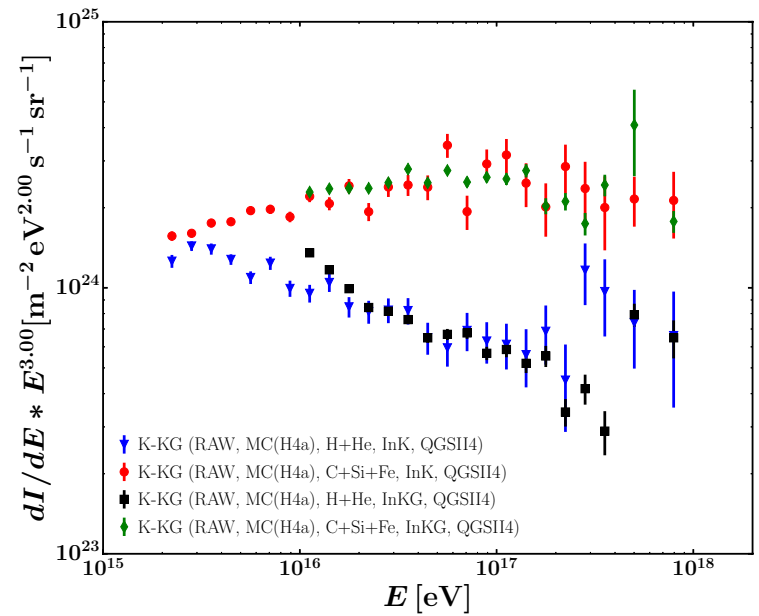

Figure 7. The reconstructed spectra for the heavy and light component are shown for data simulated using QGSJetII4 as the hadronic interaction model and $\mathrm{H} 4 \mathrm{a}$ as the astrophysical model. The spectra have been obtained with the combined reconstruction, however, separately for the two data sets defined by the core being located in KASCADE and KASCADE-Grande, respectively. In both cases the calibration based on QGSJetII4 has been used.

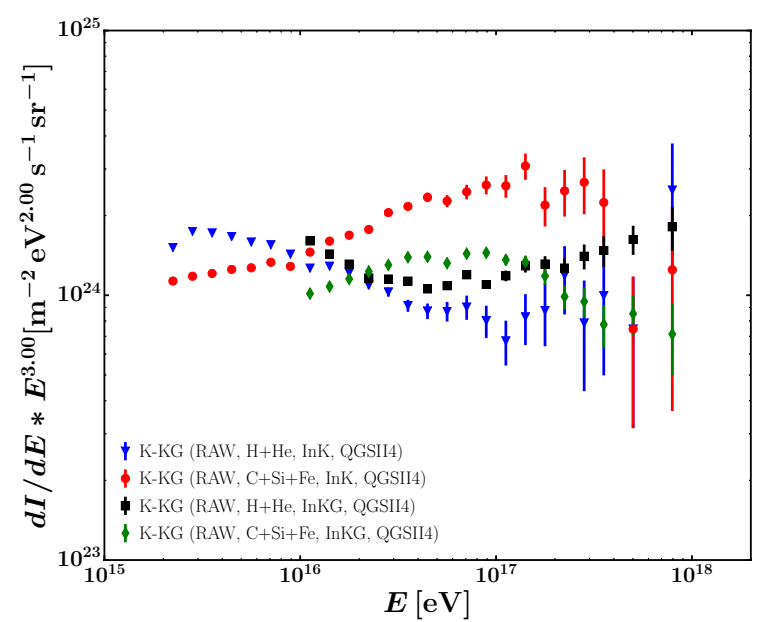

Figure 8. The reconstructed spectra for the heavy and light component are shown for measured data using calibrations based on QGSJetII4 as the hadronic interaction model. The spectra have been obtained with the combined reconstruction, however, separately for the two data sets defined by the core being located in KASCADE and KASCADE-Grande, respectively.

combined analysis now provides these spectra for an energy range of more than 3 orders of magnitude based on one unique coherent analysis. Considering the QGSJetbased results the spectra confirms the observation of all features found earlier, i.e. the light and heavy knees at approximately 3 and $100 \mathrm{PeV}$, respectively as well as the hardening at approximately $10 \mathrm{PeV}$ and the light ankle at approx. $100 \mathrm{PeV}$. This is also true for the results based on EPOS-LHC (Fig. 6) and SIBYLL 2.3 (not shown). However, again, also for the post-LHC models the relative abundances of the light and heavy generated spectra differ quite significantly from model to model.

It is interesting to note, that comparing EPOS-LHC with QGSJet-II 04 the spectra of the light component agrees quite well, but not those of heavy primaries. This

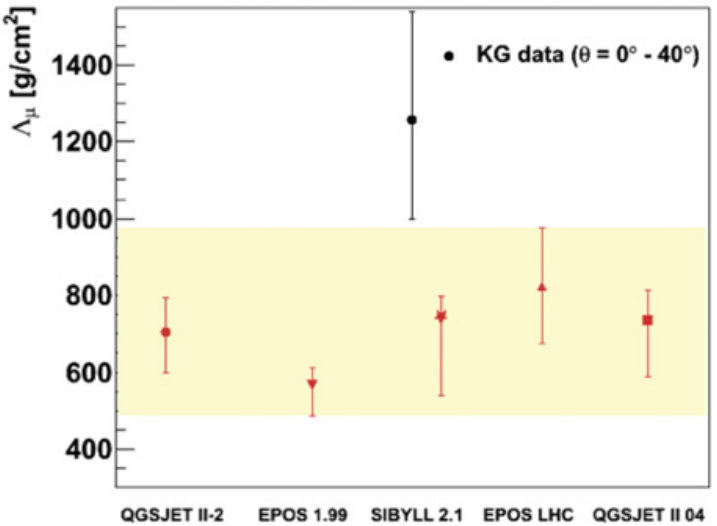

Figure 9. Muon attenuation lenghts for KASCADE-Grande in comparison with those obtained by detailed Monte-Carlo simulations based on various hadronic interaction models [27].

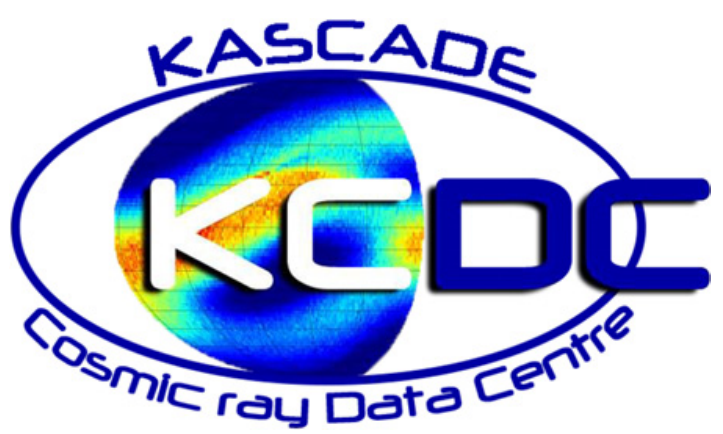

Figure 10. Logo of KCDC. The link to KCDC is: https:// kcdc.ikp.kit.edu.

can be interpreted as a hint that proton-proton interactions are better described in the post-LHC models than nucleusnucleus interactions. This is understandable as taking the LHC-data into account for the latter one an additional extrapolation or theoretical prediction is necessary to build the model.

To learn more about the source of the differences in interpreting the same data with different hadronic interaction models we deepened the analysis in the following way: For each interaction model separately a set of data was generated with the true, i.e. simulated spectra of the five elements $\mathrm{H}, \mathrm{He}, \mathrm{C}, \mathrm{Si}$, and Fe corresponding to the predictions of an astrophysical model which is relatively close to the obtained results, in this case the H4a [22] model provided by Tom Gaisser. In addition, the unique feature of KASCADE-Grande allows for crosschecking the results by dividing the data in one set containing events located within KASCADE (InK) and another set limited to events located in KASCADE-Grande (InKG). The advantage is that in one of the samples we obtain the muon number by measuring muons close to the shower core, for the other sample by measuring muons far (300-700 m distance) from the shower core. Other shower parameters are obtained with similar systematics in both samples.

The purpose of this procedure is to see how the three hadronic interaction models QGSJet-II-04 [23,24], EPOSLHC [25], and Sibyll 2.3 [26] compare to one another and to the data. 


\section{KASCADE timeline \\ (a)}

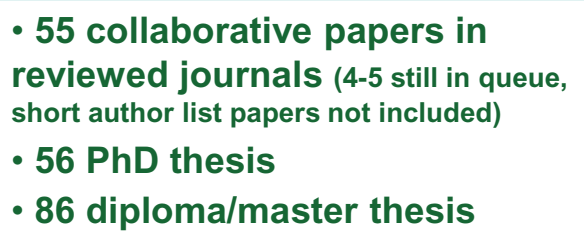

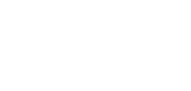

(1)

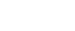

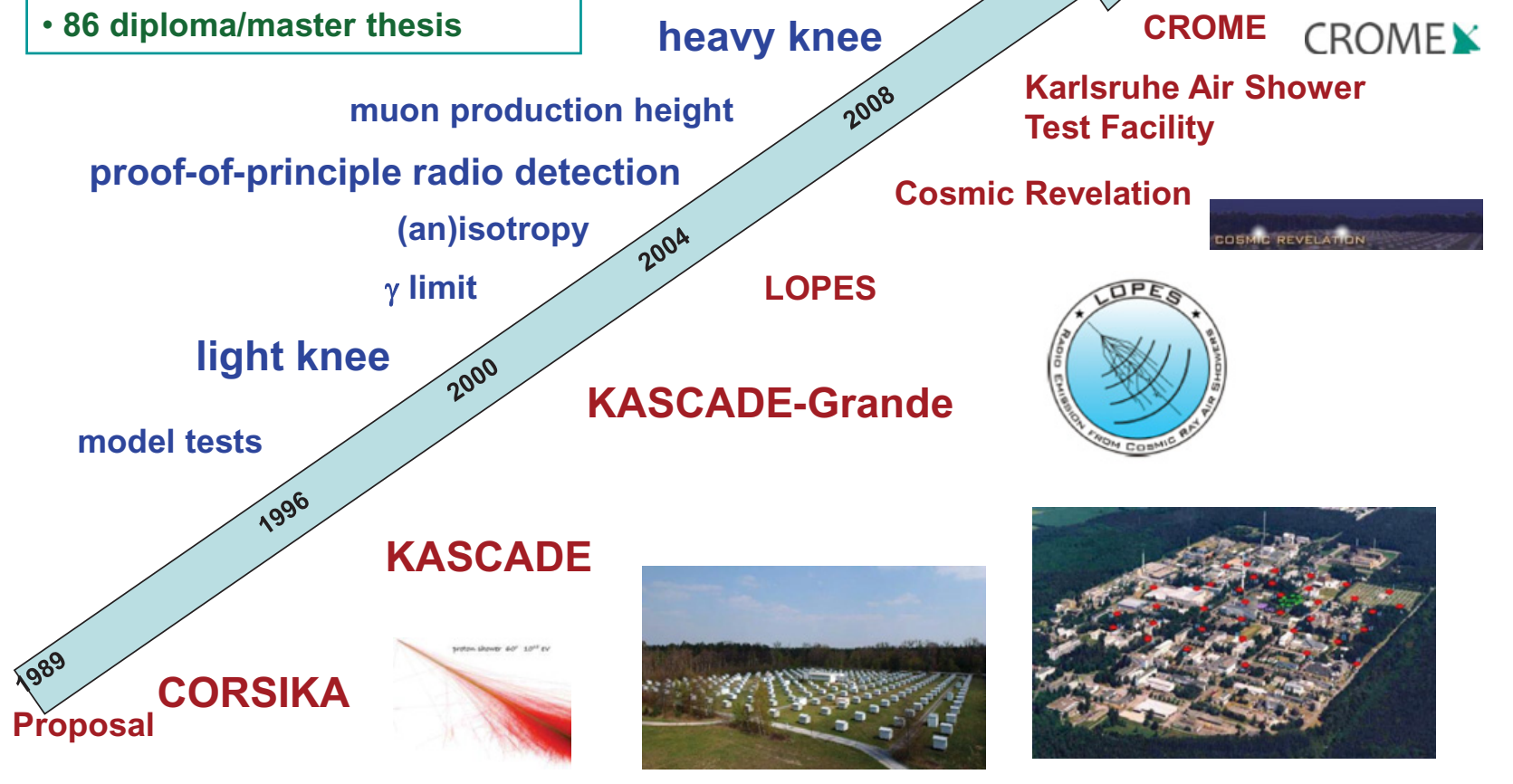

Figure 11. Timeline of the KASCADE(-Grande) facility. Shown are below the arrow of time in red the various hardware related parts of the facility and above the arrow in blue some highlights of physics results. 'Cosmic Revelation' was an art \& science project at KASCADE in 2009 [29], which had a revival in summer 2016 (see http://www . imachination.net/cosmicrevelation).

If we now apply the full (including the detector response) simulated data to the above mentioned reconstruction we expect to get back the right answer, i.e. the input spectra for both event samples. This worked within the systematic uncertainties for all three studied interaction models (see as an example Fig. 7 for QGSJet-II 04) proving an internal consistency of the models, at least. Applying the reconstruction to the two measured samples, independently, the spectra show significant differences. As an example this is shown in case of the QGSJet-II-04 model (but we found similar differences for the other two models) in Fig. 8. It is obvious that a systematic deficiency appears for the model to describe the muon content of the showers consistently. There are either too few muons predicted in the center of the EAS or too few at larger distances (i.e. the lateral distribution of muons is predicted to be too flat). The presented investigation is neither sensitive to the total number of muons (this would slightly change the energy scale predicted by the model, only) nor to give a statement of which of these two cases is more probable. But, these are strong hints that there are deficiencies in describing the slope of the lateral distribution of muons in air showers.

Another analysis studies the attenuation of muons in the Earths' atmosphere [27]. This is especially interesting for cross-checking hadronic interaction models and confirms that there are deficiencies in describing the muon production in extensive air showers at a primary energy around $100 \mathrm{PeV}$. It is based on the idea of constantintensity that assumes a flux of CRs that is isotropic in zenith angle. The number of muons measured for a certain flux is decreasing with increasing zenith angle. Therefore, the attenuation of muons in the atmosphere can be obtained (Fig. 9), where it is clear that none of the five models studied describes the observed attenuation length. The analysis was performed for the events reconstructed by the Grande stand-alone method for an event sample with a certain range of distance to the shower core and primary energy. A more detailed analysis of the muon attenuation will be published soon.

\section{The KASCADE Cosmic-ray Data Center: KCDC}

The KASCADE facility operated several large-area detectors for the measurement of EAS for more than 20 years. The major goal of KCDC [28] is the installation and establishment of a public data centre for high-energy astroparticle physics. In the research field of astroparticle physics, such a data release is a novelty, whereas the data publication in astronomy has been established for a long time. Therefore, there are no completed concepts, how the data can be treated/processed so that they are reasonably usable outside the collaboration. The project faces thereby open questions, e.g. how to ensure a consistent calibration, how to deal with data filtering and how to provide the data in a portable format as well as how a sustainable 
storage solution can be implemented. In addition, access rights and license policy play a major role and are considered.

Already with the first releases, KCDC fulfills three basic requirements needed to address the general public: KCDC as a data provider: There is free and unlimited open access to KASCADE cosmic-ray data, where a selection of fully calibrated and reconstructed quantities per individual air shower is provided. The access has to rely on a reliable data source with a guaranteed data quality.

KCDC as an information platform: For a meaningful usage of KCDC, a detailed experiment description as well as sufficient meta information on the provided data is needed for any kind of data analysis. This is accompanied by a reasonable description of the physics background as well as tutorials, which are focused on a level for teachers and pupils.

KCDC as a long-term digital data archive: To constitute a sustainable piece of work, KCDC also serves as an archive of software and data for the collaboration as well as for the public.

KCDC has started to make the data from the KASCADE experiment available to the community, where the platform to external users is already released [28] and meanwhile several times updated. Interested colleagues are invited to visit KCDC under https://kcdc.ikp.kit.edu (Fig. 10).

\section{Summary}

In summary, after separating the KASCADE-Grande measured events into light and heavy components, kneelike features are identified in the spectrum of the light component by KASCADE and of the heavy component by Grande, as well as an ankle-like feature is observed in the spectrum of the light component. Whereas the 'heavyknee' occurs at an energy where the rigidity dependent knee of the iron component is expected, the 'light-ankle' might indicate an early transition from galactic to an extragalactic origin of cosmic rays.

Whereas general structures observed in the all-particle spectrum as well as in the spectra of individual mass groups are meanwhile established and credible, the validity of the hadronic interaction models is proven to be still insufficient. KASCADE-Grande presented a new approach on comparing the three post-LHC hadronic interaction models, where in general the main message is the same for all of them: While the predictions of the hadronic interaction models are becoming more and more similar, larger discrepancies between the simulated data and measurements remain, which become obvious when comparing the results obtained for events located in KASCADE to events located in KASCADE-Grande. The main difference is observed in the muonic component of the measured air-showers. This implies that the underlying lateral density function as seen in simulations differs from the one implemented in nature.

The KASCADE-Grande collaboration has started to provide the entire data set to the public within the project KCDC, the KASCADE Cosmic Ray Data Center.

Figure 11 illustrates the timeline and some highlights of 27 years activity of the KASCADE(-Grande) facility.
The authors would like to thank the members of the engineering and technical staff of the KASCADE-Grande collaboration, who contributed to the success of the experiment. The KASCADEGrande experiment is supported in Germany by the BMBF and by the 'Helmholtz Alliance for Astroparticle Physics - HAP' funded by the Initiative and Networking Fund of the Helmholtz Association, by the MIUR and INAF of Italy, the Polish Ministry of Science and Higher Education, and the Romanian Authority for Scientific Research UEFISCDI (PNII-IDEI grants 271/2011 and 17/2011). J.C.A.V. acknowledges the partial support of CONACYT (grant CB-2008/106717) and the German-Mexican bilateral collaboration grants (DAAD-CONACYT 2009-2012, 2015-2016).

\section{References}

[1] Antoni T et al. - KASCADE, NIM A 513, 490 (2003)

[2] Apel W D et al. - KASCADE-Grande, NIM A 620 , 202 (2010)

[3] H. Falcke et al. - LOPES, Nature 435, 313 (2005)

[4] F.G. Schröder et al. - LOPES, J. Phys. Conf. Ser. 632, 012102 (2015)

[5] R. Smida et al. - CROME, EPJ Web Conf. 53, 08010 (2013)

[6] R. Smida et al. - CROME, PRL 113, 221101 (2014)

[7] A. Haungs et al. - KASCADE-Grande, ICRC 2015, The Netherlands, PoS(ICRC2015)278

[8] W.-D. Apel et al. - KASCADE-Grande, Astropart. Phys. 36, 183 (2012)

[9] T. Antoni et al. - KASCADE, Astropart. Phys. 24, 1 (2005)

[10] V.V. Prosin et al. - Tunka-133, NIM A 756, 94 (2014)

[11] M.G. Aartsen et al. - IceCube, PRD 88, 042004 (2013)

[12] Apel, W.D. et al. - Tunka-Rex and LOPES Collaborations, Physics Letters B 763, 179 (2016)

[13] W.-D. Apel et al. - KASCADE-Grande, Astrop. Phys. 65, 55 (2015)

[14] W.-D. Apel et al. - KASCADE-Grande, PRL 107, 171104 (2011)

[15] W.-D. Apel et al. - KASCADE-Grande, PRD 87, 081101(R) (2013)

[16] D. Heck et al., report Forschungszentrum Karlsruhe, FZKA 6019 (1998)

[17] W.-D. Apel et al. - KASCADE-Grande, Astropart. Phys. 47, 54 (2013)

[18] W.-D. Apel et al. - KASCADE-Grande, Adv. Space Res. 53, 1456 (2014)

[19] M. Bertaina et al. - KASCADE-Grande, ICRC 2015, The Netherlands, PoS(ICRC2015)359

[20] T. Antoni et al. - KASCADE, J. Phys. G 36, 035201 (2009)

[21] S. Schoo et al. - KASCADE-Grande, ICRC 2015, The Netherlands, PoS(ICRC2015)263

[22] T. Gaisser, Astropart. Phys. 35, 801 (2012)

[23] S. Ostapchenko, PRD 83, 014018 (2011)

[24] S. Ostapchenko, EPJ web conf. 52, 02001 (2013)

[25] T. Pierog et al., Phys. Rev. C 92, 034906 (2015)

[26] R. Engel, ICRC 1999 415, 905 (1999)

[27] J.C. Arteaga et al. - KASCADE-Grande, ICRC 2015, The Netherlands, PoS(ICRC2015)314

[28] S. Schoo et al. - KASCADE-Grande, ICRC 2015, The Netherlands, PoS(ICRC2015)262

[29] T.O. Roth and A. Haungs, Transactions, Leonardo 42, 3 (2009) 